\title{
Estudio de la pareja infértil en el Hospital Infantil Universitario Lorencita Villegas de Santos
}

\author{
Germán Barón Castañeda, MD*; María Cristina Motta**
}

\begin{abstract}
RESUMEN: La infertilidad, entendida como un año de relaciones no protegidas sin lograr la concepción, es un trastorno frecuente que puede presentarse entre un 10 y un $\mathbf{2 0 \%}$ de las parejas. Son múltiples los factores que intervienen en su génesis y que requieren diversos manejos. Se revisan 92 parejas con diagnóstico de infertilidad que han asistido a la consulta de la Unidad de Investigación Clínica en Reproducción Humana del Hospital Infantil "Lorencita Villegas de Santos". Se encuentra que tanto los casos de infertilidad primaria como de secundaria corresponden cada uno a un $50 \%$. La edad promedio de la mujer fue de $30.7 \pm 4.76$ años y la del hombre $33.1 \pm 5.87$. El tiempo de evolución de la infertilidad de $3.53 \pm \mathbf{2 . 6}$ años. Los factores encontrados como causantes de la infertilidad fueron: masculino en el $40.2 \%$, tubo-peritoneal en el $44.6 \%$, ovulatorio en $34.8 \%$, inmunológico $23.9 \%$, endometriosis $20.6 \%$, uterino $17.4 \%$ y cervical $5.43 \%$. La tasa de deserción es elevada, del $51 \%$, problema frecuente de los diversos centros de infertilidad del país. Con los diferentes tipos de tratamiento se logró una tasa de embarazos del $50 \%$, terminando en forma respectiva $5.4 \%$ en abortos y embarazos ectópicos. La tasa de embarazos posterior a microcirugía es del $65 \%$, presentándose apenas un $5 \%$ de embarazos ectópicos. Con inseminación intrauterina se obtuvo una tasa de embarazos de $\mathbf{7 6 . 5 \%}$, llegando de ellos sólo el $\mathbf{4 7 . 1 \%}$ al término. Se concluye que con un diagnóstico y manejo adecuados se puede brindar ayuda a parejas de escasos recursos económicos. Se destaca la importancia del factor tubo-peritoneal, posiblemente debido a la alta incidencia de enfermedades de transmisión sexual y por tratarse de un centro de remisión para cirugía tubárica.
\end{abstract}

PALABRAS CLAVES: Infertilidad, factores, microcirugía, inducción de ovulación, inseminación.

SUMMARY: Infertility, understood as one year of sexual unprotected intercourse without conception, is a frecuent problem that can appear in 10 to $20 \%$ of the population. Multiple factors are compromised that require different types of management. This study includes 92 couples assisting to the Unit for Clinical Investigation in Human Reproduction at Hospital Infantil "Lorencita Villegas de Santos". We found the same incidence for primary and secondary infertility, each one accounting for $50 \%$. Women were $30.7 \pm 4.76$ years old and men $33.1 \pm 5.87$. The mean evolution of infertility before diagnosis was $3.53 \pm 2.6$ years. The factors found involved as causing infertility were male factor in $\mathbf{4 0 . 2 \%}$, pelvic disorders in $44.6 \%$, ovulatory disorders in $34.8 \%$, immunologic causes in $23.9 \%$, endometriosis in $20.6 \%$, uterine factors in $17.4 \%$ and cervical disorders in $5.43 \%$. As in other centers in our country there is a large group of couples that abandon the program, $51 \%$. With the different kinds of treatment the pregnancy rate obtained is $50 \%$, with an incidence of $5.4 \%$ of abortion and ectopic pregnancies, respectively. The pregnancy rate in patients treated by microsurgery is $65 \%$, with only $5 \%$ of ectopic pregnancies. The pregnancies obtained with intrauterine insemination is $76.5 \%$, but only $47.1 \%$ of them finished in term-delivered pregnancies. We conclude that with an appropriate diagnosis and treatment good results can be obtained in couples with low socio-economical status. Noteworthy is the high incidence of pelvic factors, presumably for the importance of sexually transmitted diseases in our country and for being a center where patients are submitted for tubal surgery.

KEY WORDS: Infertility, factors, microsurgery, ovulation induction, insemination.

\section{Introducción}

En nuestro medio las dificultades en la función reproductiva constituyen un motivo cada vez más frecuente de consulta de la Unidad de Investigación Clínica en Reproducción Humana, motivo por el cual el Departamento de Ginecología y Obstetricia planteó la necesidad de llevar a cabo la revisión de los casos estudiados y manejados en el servicio que aparecen registrados como

Coordinador Unidad de Investigación. Clínica en Reproducción Humana. Hospital Infantil "Lorencita Villegas de Santos". Coordinador de Postgrado Departamento de Ginecología y Obstetricia del Hospital Infantil "Lorencita Villegas de Santos". Instructor Asociado Facultad de Medicina Colegio Mayor del Rosario.
Infertilidad, para establecer las características epidemiológicas de estas parejas, los factores etiológicos desencadenantes, los recursos diagnósticos empleados en el estudio de las mismas, los tratamientos instaurados y los resultados obtenidos.

El estudio de la pareja infértil requiere una metodología clínica y paraclínica muy cuidadosa que permita su investigación integral para poder llegar a conclusiones precisas a corto plazo y así facilitar la prescripción de un tratamiento adecuado.

Se ha calculado que la tasa de embarazo por ciclo menstrual de una pareja joven y sana, con relaciones sexuales frecuentes y sin medidas anticonceptivas es de sólo el $45 \%$, de los cuales $25 \%$ son gestaciones viables, el $10 \%$ se pierden de forma inaparente y el otro $10 \%$ en forma de abortos clínicamente diagnosticados. Solamen- 
te el $50 \%$ de las parejas logra el embarazo en los primeros seis meses y sólo el $80 \%$ durante el primer año de actividad sexual no protegida.

La tasa de infertilidad está directamente relacionada con numerosos factores epidemiológicos por lo que varía de manera significativa en cada comunidad, grupo étnico, social, etc. Dentro de los numerosos factores involucrados aparecen dentro de la literatura como más destacados los siguientes:

- Edad. Se considera como la edad más apta para la función reproductiva el período comprendido entre los 20 a 30 años de vida, después del cual la efectividad reproductiva tiene su descenso. La tasa de embarazo a los 31 años es del $74 \%$, de los 31 a los 35 desciende al $62 \%$, y en las parejas mayores de 35 años es apenas del 54\%. El riesgo de pérdidas espontáneas también aumenta con la edad, teniendo que el $12 \%$ de las mismas ocurren en mujeres alrededor de los 20 años, el $26 \%$ en mujeres de 30 a 40 años y el $75 \%$ en mujeres mayores de 40 años.

Aunque en la literatura se hace referencia al efecto de la edad de la mujer en la función reproductiva, la edad del hombre a medida que aumenta produce disminución en la cantidad y calidad del esperma aumenta el riesgo de generar alteraciones cromosómicas como por ejemplo las trisomías.

Los investigadores en salud reproductiva han descrito una significativa reducción de las tasas de embarazo por fertilización in vitro con oocitos obtenidos de pacientes de edad avanzada dependiendo de la calidad de los oocitos que aumenta el índice de pérdida del $14 \%$ en pacientes jóvenes a $45 \%$ de donantes entre los 35 y 40 años.

- Enfermedades de transmisión sexual: Enfermedades como las producidas por Gonococo, Chlamidia y Micoplasma, relacionadas directamente con los hábitos sexuales de la pareja, se describen cada vez más como factor etiológico de la infertilidad, hecho demostrado en el estudio de sociedades exclusivamente monogámicas donde los problemas de infertilidad llegan a tasas tan bajas como el 1 y $2 \%$.

- Tóxicos: El consumo de algunas sustancias produce alteraciones en la gametogénesis como aumento en la incidencia de abortos o malformaciones congénitas. Dentro de las más frecuentes se encuentran el etildietibestrol vinculado con infertilidad primaria y malformaciones del tracto reproductor en fetos femeninos, la marihuana inhibe la secreción de GnRH y puede suprimir la función reproductiva, la cocaína disminuye la espermatogénesis produciendo también alteraciones en la motilidad de los espermatozoides y el tabaco que disminuye las tasas de fertilidad especialmente si se trata de un inicio temprano o una exposición prolongada.

- Métodos contraceptivos: El desarrollo académico y laboral de la mujer en la sociedad de hoy, ha hecho que las parejas pospongan el inicio de la procreación valiéndose de métodos anticonceptivos, algunos de los cuales se han vinculado en el desarrollo de problemas que generan infertilidad. El dispositivo intrauterino (DIU) aparece como causante del $16 \%$ del factor tuboperitoneal, relacionado directamente con el antecedente de Enfermedad pélvica inflamatoria (EPI), sucedida posterior a la inserción.
- Ansiedad: El estrés se ha relacionado con disfunción hipotalámica que conduce a disfunciones sexuales de pareja como la oligoastenozoospermia en el hombre, trastornos del ciclo menstrual y anovulación en la mujer. La infertilidad por sí misma se convierte en factor generador de ansiedad lo que empeora el problema.

- Peso corporal. El control dietético y el ejercicio pueden producir disfunciones reproductivas representadas en infertilidad inexplicada o secundaria a anovulación y defectos de la fase lútea. La deprivación nutricional por la práctica de dietas con disminución irracional de la ingesta proteico calórica, ha hecho que los problemas reproductivos asociados a la baja de peso estén en aumento. Los casos extremos representados por la Anorexia nerviosa y la Bulimia juegan un papel importante en los trastornos endocrinos relacionados con problemas reproductivos por alterar el metabolismo de las endorfinas que puede regular la prolactina y la producción de gonadotropinas. La disminución de grasa corporal trae como consecuencia una baja aromatización periférica de andrógenos a estrógenos lo que aumenta el estado hipoestrogénico de estas mujeres.

La evaluación diagnóstica de la infertilidad debe ser metódica e iniciarse lo más rápido posible. Se debe realizar con una entrevista inicial, un examen físico a la pareja y con base en un diagnóstico clínico, orientar la investigación para establecer el o los factores desencadenantes de la falla reproductiva. De las pruebas que se realizan a todas las parejas en estudio de infertilidad en la Unidad de Investigación Clínica en Reproducción Humana debemos mencionar la TSH, la prolactina, la curva de LH, la curva de temperatura basal, el test de moco, el espermograma y laparoscopia como pilares diagnósticos.

La anamnesis debe recordar las características de los ciclos menstruales, embarazos previos, métodos contraceptivos, características de las relaciones sexuales, enfermedades y cirugías pélvicas previas, consumo de tóxicos, antecedentes familiares de infertilidad y trastornos endocrinos. Durante el examen físico se debe poner especial atención a los sistemas reproductor y endocrino, buscando signos que hagan sospechar trastornos o patología específica como tiroides palpable, exoftalmos, galactorrea, hirsutismo, clitoromegalia, anormalidades en útero y/o anexos, etc.

En el hombre se realiza también la anamnesis, examen físico sin olvidar investigar enfermedades y procedimientos relacionados con los genitales como enfermedades venéreas, orquitis, herniorrafías, exposición al calor, radiación, consumo de tóxicos y tabaquismo.

Con la historia y el examen físico de la pareja usualmente se puede formular una hipótesis sobre la o las causas desencadenantes de la infertilidad, y orientar las pruebas diagnósticas a fin de corroborarlas sin por esto descuidar la investigación de otros posibles factores.

\section{Materiales y métodos}

Para el presente estudio, que es de tipo retrospectivo, se escogieron las historias de las pacientes que acudieron a la consulta de infertilidad de la Unidad de Investigación Clínica en Reproducción Humana del Hospital Infantil 
Universitario "Lorencita Villegas de Santos" durante 1993 y 1994. En las 295 historias clínicas que correspondían al diagnóstico de infertilidad se evaluaron los siguientes parámetros:

\section{- Edad}

- Tipo de infertilidad: primaria o secundaria

- Tiempo de evolución

- Factores desencadenantes: ovulatorio, tubo-peritoneal, uterino, cervical, masculino, endometriosis, inmune.

- Tratamientos: suplencia tiroidea, bromocriptina, inductores de ovulación, inseminación intrauterina, microcirugía, tratamiento para endometriosis, terapia inmunológica.

- Resultados

\section{Resultados}

De las 295 parejas que iniciaron el estudio de infertilidad, sólo 92 lo han concluido, motivo por el cual éstas son las utilizadas para el análisis. Un gran porcentaje de las parejas fueron excluidas por abandono del estudio $(51.8 \%)$. 46 casos $(50 \%)$ fueron clasificados como infertilidad primaria, mientras que los 46 casos restantes (50\%) correspondieron a infertilidad secundaria.

La edad materna promedio fue de $30.7 \pm 4.76$ años con un rango entre 19 y 40 años. Por su parte la edad paterna promedio fue de $33.1 \pm 5.87$ años con un rango entre 22 y 54 años. La evolución de la infertilidad tuvo un promedio de $3.53 \pm 2.6$ años con un rango de 1 a 16 años.

El factor masculino, aislado o asociado con patología de la mujer, fue el causal de la infertilidad en 37 de los 92 casos $(40.2 \%)$. Dentro de los trastornos femeninos vale la pena destacar la importancia del factor tubo-peritoneal presente en 41 mujeres, lo cual corresponde al 44.6\%, cifra que está bastante por encima de lo reportado en la literatura. Le siguen en orden de frecuencia el factor ovulatorio en 32 pacientes ( $34.8 \%$ ), los trastornos inmunes en 22 (23.9\%), la endometriosis en 19 (20.6\%), el uterino en $16(17.4 \%)$ y por último el cervical en 5 mujeres $(5.43 \%)$. (Figura 1).

Figura 1

\section{FACTORES DE INFERTILIDAD}

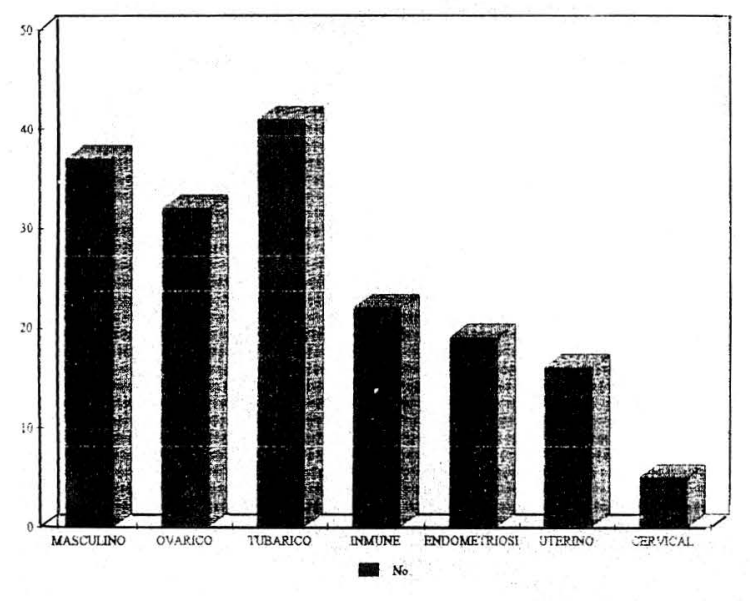

Vale la pena destacar que es infrecuente encontrar factores aislados como desencadenantes del trastorno. Prueba de ello es que asilados sólo se evidenció factor ovulatorio en 6 pacientes, tubárico en 3 y endometriosis en 4 mujeres; el factor masculino asilado sólo estuvo presente en 3 pacientes. Las asociaciones más frecuentes fueron entre los factores ovulatorio y masculino en 14 parejas (15.2\%) y tubárico y masculino en 11 (11.96\%). En general la asociación de factores masculino y femenino se presentó en 34 parejas, lo cual corresponde al 37\%, similar a lo reportado por otros grupos.

Después de realizar un estudio escalonado de los diversos factores se enfoca el tratamiento de acuerdo a los hallazgos. Hay manejos básicos como la suplencia tiroidea y el manejo de la hiperprolactinemia cuando los casos lo ameritan. Las pacientes con diagnóstico de endometriosis mínima o leve no reciben manejo específico para esta entidad, sino se corrigieron los otros factores desencadenantes.

En pacientes con factor tubo-peritoneal se practicó microcirugía por laparotomía, siendo las intervenciones más frecuentes la recanalización tubárica posterior a ligadura y la neosalpingostomía con adhesiolisis.

Tanto el factor masculino como el cervical se han manejado con inseminación intrauterina con semen capacitado; para este fin se ha utilizado el método de migración-sedimentación. Con frecuencia en el trastorno masculino se encuentra la asociación de espermatozoides aglutinados con leucospermia, casos en los cuales se asocia la antibióticoterapia con doxiciclina.

El esquema de inducción de ovulación utilizado se basa en el citrato de clomifeno, en dosis de $50 \mathrm{mg}$ diarios del día 5 al 9 del ciclo. Se utiliza en esta forma para disminuir los riesgos de hiperestimulación ovárica y embarazos múltiples. En la mayoría de pacientes se ha logrado la ovulación, dato confirmado por el hallazgo ecográfico. En algunos casos se asocian 5.000 UI de gonadotropina coriónica para lograr efecto de LH.

Las alteraciones inmunes no sólo se presentan en mujeres con pérdida recurrente del embarazo, hecho demostrado por la alta incidencia observada en este grupo de pacientes. Con frecuencia en la infertilidad que antiguamente se describía como "de causa no explicada" se puede encontrar patología aloinmune por lo cual se recomienda la inmunoterapia con linfocitos paternos.

Con este enfoque diagnóstico y terapéutico, en las 92 parejas presentadas se han obtenido 46 embarazos (50\%). De ellos, 36 han terminado en parto (39.1\%). Las tasas de aborto y embarazo ectópico son iguales, presentándose cada una de estas entidades en 5 pacientes (5.4\%). (Tabla 1).

Vale la pena destacar que el resultado obtenido con microcirugía es bastante satisfactorio. De 20 pacientes sometidas a intervención quirúrgica, 13 han quedado embarazadas $(65 \%)$, con una sola gestación siendo ectópica $(5 \%)$.

De igual forma se ha obtenido un buen número de embarazos en parejas que han requerido inseminación, ya sea con semen homólogo capacitado o con semen de donante $(75 \%)$. Estos datos se encuentran resumidos en la Tabla 2. 
Tabla 1

RESULTADOS DEL TRATAMIENTO

\begin{tabular}{|lll|}
\hline Embarazos & No. & $\%$ \\
\hline A término & 36 & 39,1 \\
Aborto & 5 & 5,4 \\
Ectópico & 5 & 5,4 \\
Total & 46 & 50 \\
& & $\mathrm{n}=92$ \\
\hline
\end{tabular}

Tabla 2

\section{RESULTADOS CON MANEJOS ESPECIFICOS}

\begin{tabular}{|l|ll|ll|ll|}
\hline Embarazos & Microcirugía & Inseminación & Inducción \\
\hline \multicolumn{1}{|c|}{$\mathrm{n}$} & \multicolumn{2}{|c|}{20} & \multicolumn{2}{|c|}{17} & \multicolumn{2}{|c|}{24} \\
& $\mathrm{n}$ & $(\%)$ & $\mathrm{n}$ & $(\%)$ & $\mathrm{n}$ & $(\%)$ \\
Término & 12 & $(60 \%)$ & 8 & $(47.1 \%)$ & 13 & $54.2 \%)$ \\
Aborto & & & 3 & $(17.6 \%)$ & 2 & $(8.3 \%)$ \\
Ectópico & 1 & $(5 \%)$ & 2 & $(11.8 \%)$ & 2 & $(8.3 \%)$ \\
Total & 13 & $(65 \%)$ & 13 & $(76.5 \%)$ & 17 & $(70.8 \%)$ \\
\hline
\end{tabular}

\section{Conclusiones}

A pesar de que en nuestro medio, día a día es mayor el número de parejas que busca ayuda para solucionar los problemas que ocasionan dificultades reproductivas, el estudio muestra una altísima tasa de deserción que impide establecer en forma más exacta los factores etiológicos implicados, la aplicación de tratamientos y los resultados obtenidos para ajustarlos a las características de nuestra población.
Los promedios de edad de los miembros de las parejas estudiadas están dentro de los promedios establecidos en la literatura revisada.

El factor peritoneal aparece con una mayor incidencia que la reportada en la literatura, lo que puede ser reflejo de dos situaciones. La primera de ellas es el hecho de tratarse de un hospital centro de referencia, por lo cual hay gran número de pacientes remitidas para recanalización tubárica. La segunda es la iniciación temprana de actividad sexual con aumento de la promiscuidad, lo cual puede incrementar en forma significativa la incidencia de enfermedad pélvica inflamatoria y sus secuelas sobre la fertilidad.

Mientras que el período de tiempo considerado óptimo para iniciar el estudio de las parejas infértiles está establecido en un año, el promedio de las parejas estudiadas en el trabajo fue mayor a tres años, lo que podría disminuir el éxito del tratamiento.

El resultado obtenido con la aplicación de la técnica microquirúrgica es similar a lo descrito en la literatura y a pesar del abandono de algunas de estas parejas la tasa de embarazo encontrada es más que satisfactoria. Esto refuerza la idea que es una técnica que no debe ser abandonada, especialmente en centros donde no se dispone de tecnología de reproducción asistida como fertilización in vitro.

Los embarazos logrados después del estudio y aplicación de tratamientos enunciados, reflejan un adecuado enfoque diagnóstico y terapéutico a pesar de no contar con muchas de las técnicas de reproducción asistida y de las condiciones socioeconómicas y culturales de las parejas con falla reproductiva.

Este trabajo abre las puertas a otros estudios que permitan evaluar y analizar aspectos más específicos sobre la etiología, diagnóstico y tratamiento de las parejas infértiles de nuestro hospital.

\section{BIBLIOGRAFIA}

1. Rueda GR., Rueda SR. Generalidades, principios y definiciones en Guías diagnósticas en infertilidad. Bogotá, ACEP, 1987.

2. Di Paola G., Brocaccini JC. Enfoque de la pareja estéril. Avances en infertilidad humana. Asch/Acosta. Buenos Aires, Argentina. Ed. Panamericana, 1988.

3. Pardo G., Renohi J., Pelliecr F. Efectos de la heroína, cocaína, marihuana y metadona sobre la reproducción. Rev. Col. Obstet. Ginecol. 1986; 37(3).

4. Kendall M. La encuesta mundial de fecundidad (E.M.S.). Situación actual y resultados. Julio 1989.

5. Seibel M., Taimor M. Aspectos emocionales de la infertilidad. Fertil Steril 1982; 37: 137.

6. Moghishi KS., Sacco A. Immunologic infertility in cervical mucus and postcoital test. Am. J. Obstet. Ginecol. 1985; 136: 941.

7. Metzger DA., Hanery A. Causas de la endometriosis. Clínicas de Ginecología y Obstetricia. 1989; 1-14.

8. Soulos MR et al. Endometriosis and anovulation. Am. J. Obstet. Gynecol. 1986; 125: 412.

9. Olive DL., Lee KL. Análisis del tratamiento secuencial y los protocolos de endometriosis asociados a infertilidad. Am. J. Obstet. Gynecol. 1989; 154: 613 .
10. Muscato JJ et al. Sperma phagocitosis by human peritoneal macrophagues. Am. J. Obstet. Gynecol. 1982; 144: 503.

11. Antony AL., Tursoy RN. Evaluación del acetato de medroxiprogesterona como tratamiento en endometriosis. Obstet. Gynecol. 1988; 72: 323.

12. Barberi RL., Bryan KJ. Danazol: aplicación farmacológica y terapéutica. Am. J. Obstet. Gynecol. 1991; 141: 453.

13. Steingold KA., Cedars $M$ et al. Tratamiento de la endometriosis con análogos de GnRH. Obstet. Gynecol. 1987; 69(3): 403.

14. Pérez LE., Mendoza JC. Tratamiento de la endometriosis severa y extensiva con microcirugía y Danazol. Anales del Congreso Nacional de Obstetricia y Ginecología. Pereira 1988.

15. Rock J et al. Endometriosis: Diagnóstico e implicaciones en los noventa. Am. J. Obstet. Gynecol. 1991; 128: 362.

16. Syrop CH., Harme J. Cambios cíclicos de líquido peritoneal en parejas normales e infértiles. Obstet. Gynecol. 1987; 69: 416.

17. Speroff L., Glass RH., Kase NG. Female infertility en Clinical gynecologic endocrinology and infertility. $5^{\text {a }}$ Edition. Williams \& Wilkins. 1994; 26: 809. 